\title{
Extracorporeal membrane oxygenation rescue for extrinsic airway compression and cardiac tamponade with multiple transports for mediastinal Burkitt's lymphoma radiation and chemotherapy: Case report and review
}

\author{
Vincent Lau*1, John Miller², Dennis Modry 2,3, Gurmeet Singh"3,4 \\ ${ }^{1}$ University of Alberta, Faculty of Internal Medicine, Edmonton, AB, Canada \\ ${ }^{2}$ Department of Clinical Perfusion Services, Mazankowski Alberta Heart Institute, Alberta Health Services, University of Alberta, \\ Edmonton, AB, Canada \\ ${ }^{3}$ Division of Cardiac Surgery, University of Alberta, Edmonton, AB, Canada \\ ${ }^{4}$ Division of Critical Care Medicine, University of Alberta, Edmonton, AB, Canada
}

Received: October 21, 2015

DOI: $10.5430 /$ css.v2n1p56
Accepted: November 15, $2015 \quad$ Online Published: November 17, 2015

URL: http://dx.doi.org/10.5430/css.v2n1p56

\begin{abstract}
A 24-year-old male presented with respiratory failure and superior vena cava (SVC) syndrome secondary to Burkitt's lymphoma with mediastinal mass causing tracheobronchial compression. Femoral-femoral veno-arterial (VA) extracorporeal membrane oxygenation (ECMO) was initiated for hypercarbic and hypoxemic respiratory failure as well as cardiac tamponade. Multiple off-site transports to and from the cancer institute for chemotherapy and fractionated external beam radiation were required. Resultant tumor shrinkage allowed ECMO weaning and decannulation. The patient was discharged and returned to post-graduate education. Aggressive intervention with ECMO support permitted chemotherapy, radiation treatment, and ultimately a successful outcome in high-grade Non-Hodgkin's lymphoma (NHL), specifically if the respiratory failure is secondary only to extrinsic airway compression.
\end{abstract}

Key Words: Extracorporeal membrane oxygenation, Malignancy, Mediastinal, Transport, Lymphoma

\section{INTRODUCTION}

Extracorporeal membrane oxygenation (ECMO) for cardiac and respiratory failure is a well-established support modality, and has been described for rescue in respiratory failure in hematologic malignancies. ${ }^{[1,2]}$ Usage in primary mediastinal malignancies causing cardiorespiratory collapse (i.e. tam- ponade), however, is not well-described. Non-Hodgkin's lymphomas (NHL) rarely present as bulky mediastinal tumors. ${ }^{[3]}$ This case presents a diagnostic and management challenge due to tumor burden from extrinsic compression to the cardiorespiratory system. Another logistical challenge was also transfer of the patient to and from the cancer insti-

\footnotetext{
*Correspondence: Vincent Lau; Email: vinceissaclau@gmail.com; Address: Mazankowski Alberta Heart Institute, 8410-112 Street, Edmonton, Alberta, T6G 2B7, Canada.
} 
tute at a different hospital with multiple life support systems in place, including ECMO. We describe the rescue usage of ECMO for respiratory failure secondary to extrinsic airway compression and cardiac tamponade from a mediastinal NHL (aggressive Burkitt's lymphoma) in a patient requiring support for concomitant radiation and chemotherapy, with tumor regression and ultimate survival and return to the community.

\section{CASE Report}

A 24-year-old male presented with a 2 month history of a progressively growing neck mass, neck and facial swelling and associated nausea and vomiting, fevers, night sweats, and a dry cough. Computed tomography (CT) scan of the chest, abdomen and pelvis showed extensive neck and anterior mediastinal adenopathy $(10.6 \mathrm{~cm} \times 9.5 \mathrm{~cm} \times 9.4 \mathrm{~cm})$ with marked superior vena cava (SVC) narrowing and posterior tracheal flattening (see Figure 1). Respiratory distress required emergent awake fiberoptic intubation in the operating theatre post-admission day one. Subsequently, a right neck lymph node biopsy was performed in the operating theatre. Ventilator support was continued post-operative in the intensive care unit. Worsening SVC syndrome with dependent plethora in the face and upper extremities developed. While awaiting tissue diagnosis from the lymph node biopsy, rapid deterioration from enlarging tumor mass resulted in hypoxemic $\left(\mathrm{PO}_{2}=50 \mathrm{mmHg}\right)$ and hypercarbic $\left(\mathrm{PCO}_{2}=\right.$ $55 \mathrm{mmHg}$ ) respiratory failure despite maximal support from conventional mechanical ventilation (Pressure control $24 \mathrm{~mm}$ $\mathrm{H}_{2} \mathrm{O}$, PEEP $15 \mathrm{~mm} \mathrm{H}_{2} \mathrm{O}, \mathrm{FiO}_{2}=100 \%$ ) on post-admission day three. With the absence of on-site thoracic surgery to perform urgent trachea-bronchial stenting, cardiac surgery and cardiovascular intensive care unit teams were consulted. The consensus was to proceed with rescue ECMO support while administering radiation and chemotherapy on postadmission day four. We preferred veno-venous (VV) ECMO for respiratory support, employing a right internal jugular, dual lumen, single cannula strategy. However, ultimately veno-arterial (VA) ECMO was selected based on bilaterally thrombosed internal jugular veins and the presence of a small pericardial effusion, which raised the specter of possible future tamponade and possible pulmonary embolism from the prior thromboses.

On post-admission day four, the patient was taken to the operating theatre again for insertion of VA-ECMO. An 8-mm Gelweave (Vascutek, Terumo, Inchinnan, Scotland) graft was anastomosed to the left femoral artery providing distal and proximal flow. 28-French and 12-French venous cannulae (Edwards Lifesciences LLC, Irvine, California, USA) were placed in the proximal and distal left common femoral vein respectively. The distal femoral venous cannula maintained

Published by Sciedu Press distal limb venous drainage. A Jostra Maquet Rotaflow Centrifugal Pump RF 32 with Quadrox iD oxygenator (Maquet Cardiopulmonary AB, Hirrlingen, Germany) completed the ECMO circuit. Five liter per minute flows was readily achieved for cardiac output through ECMO with resolution of hypoxemia. Intravenous heparin was continuously infused to prevent extracorporeal circuit thrombosis, targeting a PTT of 160-180 seconds. Immediately following cannulation, the patient was transported via specialized ambulance (chosen for its ability to transport the patient and multiple life support systems, including ECMO) to the cancer institute for radiotherapy.

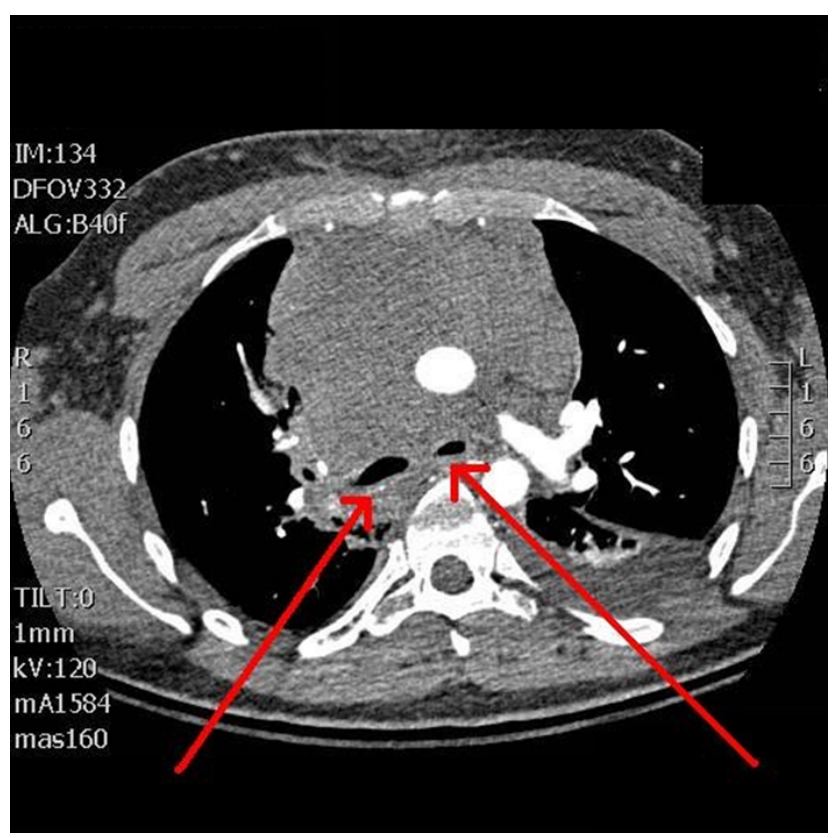

Figure 1. Initial CT chest at presentation - red arrows note bilateral bronchi compression externally from mediastinal tumor (Burkitt's lymphoma)

From post-admission day four to nine, in consultation with the hematology-oncology, the patient received 5 fractionated external beam radiotherapy treatments with transport to and from the cancer institute. Lymph node biopsy pathology confirmed Burkitt's high grade mature B-cell lymphoma. A right posterior iliac crest bone marrow biopsy demonstrated no marrow involvement. R-CHOP (rituximab, cyclophosphamide, hydroxydaunorubicin, oncovin, and prednisone) chemotherapy was started while still on VA-ECMO on postadmission day ten, as chosen by hematology-oncology for its effectiveness against high-grade Burkitt's lymphoma. The reason for the rapid institution of radiation and chemotherapy was to quickly shrink the tumor burden causing extrinsic compression, and allow for oxygenation and ventilation off ECMO and on traditional mechanical ventilation. No ECMO 
circuit complications from chemotherapy occurred. One revision of the left femoral venous cannula was required for hematoma formation and bleeding on post-admission day eight. The pericardial effusion enlarged, causing cardiac tamponade. On post-admission day ten, a pericardiotomy through a lateral thoracotomy was performed to create a pericardial window to drain the effusion into the pleural cavity and evacuate it through placement of a left sided chest tube.

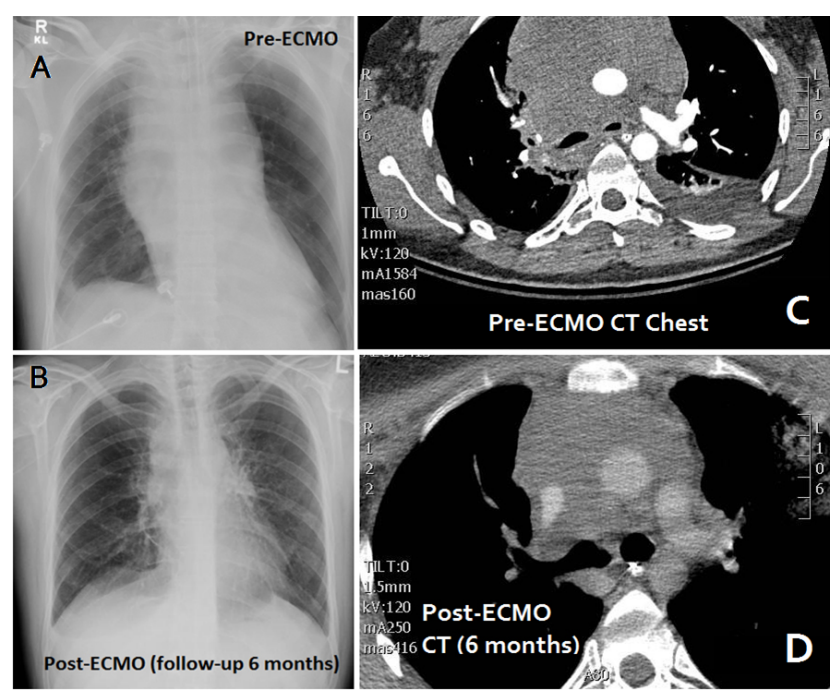

Figure 2. Pre and post-ECMO imaging

Panel A: Pre-ECMO chest X-ray; Panel B: Post-ECMO chest X-ray at 6 months; Panel C: Pre-ECMO CT Chest with mediastinal tumor measuring $10.6 \mathrm{~cm} \times 9.5 \mathrm{~cm} \times 9.4 \mathrm{~cm}$; Panel D: Post-ECMO CT chest with mediastinal tumor shrinkage to $1.9 \mathrm{~cm} \times 1.8 \mathrm{~cm} \times$ $1.3 \mathrm{~cm}$ with resolution of tracheobronchial compression

Eventually, VA-ECMO was completely weaned off and decannulated after 8 days of ECMO support (post-admission day twelve). The patient was extubated after 15 days of mechanical ventilation (post-admission day fifteen). He was transferred to the cancer institute to complete four cycles of chemotherapy (post-admission day twenty-two). Consolidation chemotherapy and subsequent autologous stemcell transplant were being entertained, but not required because of the patient's excellent response to chemotherapy and radiation already given. Decreased tumor size (shrank to $1.9 \mathrm{~cm} \times 1.8 \mathrm{~cm} \times 1.3 \mathrm{~cm})$ and mass effect on mainstem bronchi and other mediastinal tissues was confirmed by X-ray and CT at 6 months (see Figure 2). Follow-up CT/positron emission tomography (PET) scan at 6 months showed regression of the previous Burkitt's lymphoma with no residual tumor activity (see Figure 3) - negative for fludeoxyglucose (FDG) activity. Unfortunately, a pre-ECMO PET scan was not performed for comparison. The patient has been discharged from the cancer institute and continues to do well without need for further chemotherapy or radiation, having returned to post-graduate education at 2 year follow-up.

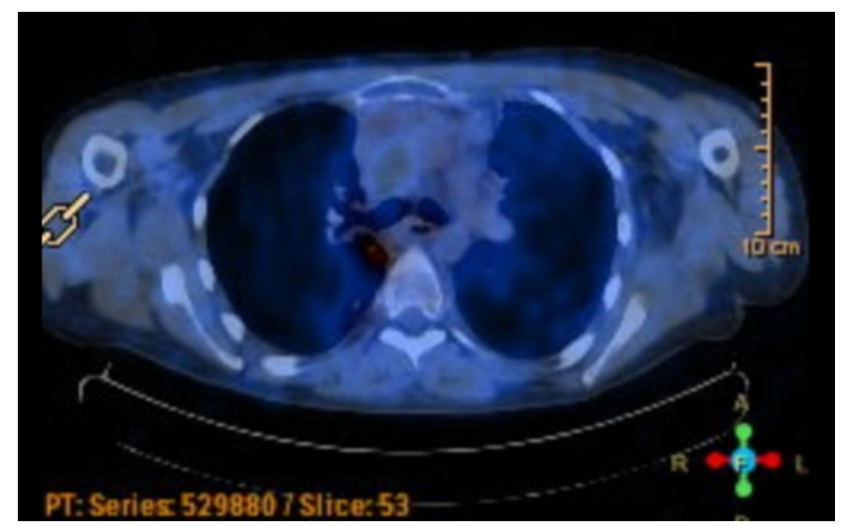

Figure 3. Follow-up PET/CT of the chest showing no FDG activity (orange color) at 6 months- resolution of Burkitt's lymphoma of the mediastinum

\section{DisCUSSION}

Mediastinal NHL presenting as a solitary bulky tumor is relatively rare, ${ }^{[3]}$ although thoracic and mediastinal lymphadenopathy is not. Main-stay of treatment is external beam radiation and chemotherapy, in addition to immunologic therapy like rituximab. ${ }^{[4]}$ Airway compression is seen in only $5 \%$ of NHL cases but can lead to respiratory failure requiring mechanical ventilation. Although bronchoscopic stenting remains the treatment of choice in external compression bronchial collapse, ${ }^{[5]}$ rapid deterioration and absence of onsite thoracic surgery made this nonviable in this case. ECMO is a viable support modality in the management of mediastinal malignancy, prior to radiation/chemotherapy or debulking surgery in both pediatric and adult populations. ${ }^{[1,6-12]}$ Primary mediastinal tumors should be considered treatable until proven otherwise, while metastases from known primaries should be considered on an individual case-by-case basis. ${ }^{[10]}$

A review of the literature shows the combined number of adult (age $\geq 18$ years old) cases with hematologic malignancies and rescue ECMO for respiratory failure to be 20 patients, with a survival of 12 patients to hospital discharge $(60 \%){ }^{[1,6-12]}$ Wohlfarth et al. reports the largest case series hematological malignancies treated with concomitant ECMO for respiratory failure of 14 patients with an overall retrospective study survival of $50 \%$ (7 of 14 patients). ${ }^{[1]}$ However, 15 patients $(75 \%)$ in this combined cohort of 20 patients requiring ECMO were for complications due to treatment of the malignancy (i.e. pneumonia, acute respiratory distress syndrome [ARDS], transfusion associated lung injury [TRALI], abdominal sepsis), and not to the extrinsic compression of the mediastinal tumor itself. ${ }^{[1-4,11,12]}$ In fact, only 5 patients with purely external airway compression necessitated ECMO, 
and all of these patients survived $(100 \%) .^{[1,2,9,10,12]}$ This is similar to our case report patient, who had no intrinsic lung injury complications (ARDS, TRALI, pneumonia) from his mediastinal malignancy and only had extrinsic compression as the cause of his respiratory failure.

VA rather than VV-ECMO in this case was chosen based on vascular access and in anticipation of potential future cardiac deterioration from tamponade physiology and possible future pulmonary emboli. Right internal jugular single cannula dual-lumen venous cannulation would normally have been preferred; ${ }^{[6]}$ however, bilateral internal jugular venous thrombosis necessitated femoral access. Additionally, a concomitant pericardial effusion caused eventual pericardial tamponade, requiring pericardiotomy. This case is unique as it is the first in the literature to describe rescue VA-ECMO in patient with combined respiratory failure from extrinsic compression, as well as cardiac tamponade causing cardiovascular collapse. It is also unique for multiple VA-ECMO safe transports to and from another institution for specialized oncologic therapy, and the durability of the ECMO circuit and the patient to withstand these multiple trips in an ambulance with all life support systems intact. Despite the logistical challenges posed, no significant complication occurred during treatment, other than a femoral venous cannulation site hematoma requiring surgical revision.

\section{Conclusions}

ECMO support for external mediastinal tumor tracheobronchial compression can successfully provide support when conventional mechanical ventilation fails. We believe this is the first case in the literature of VA-ECMO usage for the concomitant respiratory and cardiovascular collapse from tamponade physiology. ECMO instituted for respiratory failure secondary to extrinsic airway compression seems to carry a better prognosis than those instituted for complications of radiation and chemotherapy (i.e. pneumonia, ARDS, TRALI) treatment for hematological malignancies. Both radiation and chemotherapy can safely be instituted while on ECMO. Furthermore, the ECMO circuit itself is also durable to be transported on multiple occasions to different institutions.

\section{REFERENCES}

[1] Wohlfarth P, Ullrich R, Staudinger T, et al. Extracorporeal membrane oxygenation in adult patients with hematological malignancies and severe acute respiratory failure. Critical Care. 2014; 18: R20. PMid: 24443905. http://dx.doi.org/10.1186/cc13701

[2] Gorjup V, Fister M, Noc M, et al. Treatment of sepsis and ARDS with extracorporeal membrane oxygenation and interventional lung assist membrane ventilator in a patient with acute lymphoblastic leukemia. Respir Care. 2012; 57: 1178-1181. PMid: 22369998. http://dx.doi.org/10.4187/respcare.01393

[3] Cohen AJ, Thompson L, Edwards FH, et al. Primary cysts and tumors of the mediastinum. Ann Thorac Surg. 1991; 51: 378-384. http://dx.doi .org/10.1016/0003-4975(91) 90848-K

[4] Hsu PK, Hsu HS, Li AF, et al. Non-Hodgkin's lymphoma presenting as a large chest wall mass. Ann Thorac Surg. 2006; 81: 1214-128. PMid: 16564245. http://dx.doi.org/10.1016/j.athoracsu r.2005.11.044

[5] Schmidt B, Massenkeil G, John M, et al. Temporary tracheobronchial stenting in malignant lymphoma. Ann Thorac Surg. 1999; 67: 1448-1450. http://dx.doi.org/10.1016/S0003-4975(99 ) 00254-4

[6] Wu MY, Wu TI, Tseng YH, et al. The feasibility of venovenous extracorporeal life support to treat acute respiratory failure in adult cancer patients. Medicine. 2015; 94: e893. PMid: 26020399. http: //dx.doi.org/10.1097/MD.0000000000000893
[7] Huang YL, Yang MC, Huang CH, et al. Rescue of cardiopulmonary collapse in anterior mediastinal tumor. Pediatr Emergency Care. 2010; 26: 296-298. PMid: 20386416. http://dx.doi.org/10.1097/P EC. Ob013e3181d6daf 0

[8] Frey TK, Chopra A, Lin RJ, et al. A child with anterior mediastinal mass supported with veno-arterial extracorporeal membrane oxygenation. Pediatr Crit Care Med. 2006; 7: 479-481. PMid: 16885791. ht tp://dx.doi.org/10.1097/01.PCC.0000235247.10880.F8

[9] Aboud A, Marx G, Sayer H, et al. Successful treatment of an aggressive non-Hodgkin's lymphoma associated with acute respiratory insufficiency using extracorporeal membrane oxygenation. Interactive Cardiovascular and Thoracic Surgery. 2008; 7: 173-174. PMid: 18045830. http://dx.doi.org/10.1510/icvts. 2007 159921

[10] Stewart AS, Smythe WR, Aukburg S, et al. Severe acute extrinsic airway compression by mediastinal tumor successfully managed with extracorporeal membrane oxygenation. ASAIO Journal. 1998; 44(3): 219-221. PMid: 9617955. http://dx.doi.org/10.1097/00002 480-199805000-00018

[11] Liao WI, Tsai SH, Chiu SK. Successful use of extracorporeal membrane oxygenation in a hematopoietic stem cell transplant patient with idiopathic pneumonia syndrome. Respir Care. 2013; 44: 219-221. http://dx.doi.org/10.4187/respcare.01716

[12] Felten ML, Michel-Cherqui M, Puyo P, et al. Extracorporeal membrane oxygenation use for mediastinal tumor resection. Ann Thoracic Surgery. 2010; 89: 1010-1014. PMid: 20172192. http: //dx.doi.org/10.1016/j.athoracsur.2009.09.064 